\title{
Algunas Observaciones sobre Putnam Y LA JUSTIFICACIÓN RACIONAL
}

\author{
Pablo Melogno
}

\begin{abstract}
In Reason, Truth and History, Hilary Putnam characterized informal rationality as an alternative that allows to overcome limitations evidenced by the formalist conceptions of rationality. Initially, the paper reviews the characterization provided by Putnam, trying to determine what kind of statements may function as principles of rationality within an informal framework. It establishes that, while Putnam denies any universal principle of rationality, he accepts relative, general and vague principles, that remain subject to needs of cognitive interrelationship with the environment. It shows that principles make exceptions, do not have specific contents and do not provide conclusive results; however, this does not prevent us from assessing the adequacy of conflicting principles. If two individuals have different conceptual schemes, this is due to the fact that they have different cognitive purposes, and it can be asserted that the choices of both are rational. From this point, the paper defends and analyzes the possibility of reviewing principles whose denial is currently not conceivable. Finally, it states that both expressing and maintaining beliefs of controversy are historical elements that should be contemplated by an informal conception of rationality.
\end{abstract}

Keywords: Putnam; informal rationality; conceptual schemes; justification.

\section{Introducción}

En Razón, Verdad e Historia Putnam caracteriza la racionalidad informal como una alternativa que permite superar los límites de las concepciones formalistas de la racionalidad, y de las críticas al concepto de racionalidad provenientes de posturas relativistas. Esta concepción informal supone que la elección de criterios de racionalidad y la justificación de creencias son históricamente variables y están sujetos a necesidades de interrelación cognitiva con el entorno, sin que esto implique se que trate de procesos arbitrarios o infundados. Este trabajo parte de una revisión de la posición de Putnam, buscando establecer qué tipo de enunciados pueden funcionar como principios de racionalidad en un marco informal, y enfatizando que la caracterización putnamiana de la racionalidad conlleva la negación de principios algorítmicos para la justificación racional de creencias, pero no impide la defensa de otra clase de principios.

En función de esto se busca especificar algunas características posibles de lo que puede considerarse como principios informales de racionalidad. Se trata de principios relativos, en la medida en que son dependientes de necesidades cognitivas

Principia 17(3): 419-438 (2013).

Published by NEL — Epistemology and Logic Research Group, Federal University of Santa Catarina (UFSC), Brazil. 
históricamente variables, y permanecen asociados a esquemas conceptuales. Se trata asimismo de principios indeterminados, en cuanto no establecen contenidos ni condiciones concretas de aplicación, y no tienen carácter universal, ya que son revisables a la luz de principios rivales igualmente fértiles o adecuados. Esto supone que una vez que la revisión de los principios es amplia pero no ilimitada, resulta posible evaluar la adecuación y la fertilidad de principios en conflicto, si bien el resultado de la evaluación no es necesariamente unívoco ni concluyente. Por esto, si dos sujetos tienen diferentes esquemas conceptuales, ello se debe a que tienen diferentes propósitos epistémicos, y en esta medida puede afirmarse que las elecciones de ambos son racionales.

En estos términos, la posibilidad de revisión racional puede ampliarse hasta abarcar principios cuyas condiciones de revisión no resultan actualmente concebibles, en cuanto la perspectiva de Putnam permite afirmar que todo esquema conceptual incluye principios que están sustraídos del escrutinio crítico, en cuanto bajo contextos históricos concretos sus condiciones de negación resultan inconcebibles para los sujetos que los sostienen. A efectos de clarificar el alcance de estas formulaciones, se compara la posición de Putnam en Razón, verdad e historia, de 1981, respecto de Las mil caras del realismo de 1987, en cuanto en el primero de estos trabajos Putnam sostiene que algunos principios mínimos de la racionalidad son intrínsecamente irrevisables, mientras que en el segundo afirma que todo enunciado con pretensiones de racionalidad debe estar abierto a revisión crítica.

A modo de conclusión, se defiende que un forma interesante de reconstruir la racionalidad informal putnamiana consiste en moderar las pretensiones de identificar principios absolutamente a priori o intrínsecamente racionales. Esto resulta posible aún concediendo que en un esquema conceptual dado existen enunciados sustraídos de la crítica, pero que ello no responde a sus características intrínsecas sino a las características históricas del esquema conceptual al que pertenecen, y a las necesidades epistémicas de los sujetos que lo utilizan.

Durante la primera mitad del siglo XX, las concepciones de la racionalidad desarrolladas desde la filosofía de la ciencia en el marco del neopositivismo y el racionalismo crítico ofrecieron una forma de considerar la racionalidad forjada en torno a una serie de rasgos bien definidos. Partían de que la racionalidad científica era la racionalidad por definición, y que el considerar o no racionales a otras actividades debía ser el resultado de su nivel de ajuste a los códigos de la ciencia. Suponían que los criterios para establecer la racionalidad de una creencia o de la elección de una teoría eran universales, de validez invariante e independiente de las contingencias históricas. Mantenían asimismo la convicción de que los criterios para elegir entre teorías científicas rivales eran susceptibles de formalización lógica y aplicación relativamente mecánica.

Esta concepción de la racionalidad entrará en una crisis irreversible al acercarse

Principia 17(3): 419-438 (2013). 
la mitad del siglo XX, crisis que tendrá su punto de inflexión con la publicación de La estructura de las revoluciones científicas de Thomas Kuhn (2004). El cuestionamiento a las concepciones tradicionales de la racionalidad científica operado mediante la obra de Kuhn -entre otros- implicará sostener que los criterios a través de los que se establece la racionalidad de una creencia o la elección de una teoría son construcciones históricas, tan permeables al paso del tiempo como las creencias cuya racionalidad pretenden fundamentar. Implica también considerar que los mecanismos de elección de teorías no se agotan en la puesta en marcha de criterios de evaluación, sino que los factores sociológicos y psicológicos, tradicionalmente ubicados en el contexto de descubrimiento y excluidos del contexto de justificación, tienen un papel preponderante a la hora de determinar qué creencias consideramos como racionales.

En esta nueva imagen de la ciencia resultan centrales los problemas asociados a la elección racional entre teorías rivales, ya que si no existe una serie de criterios universales de evaluación, queda abierta la posibilidad de que cada científico justifique su elección de forma recursiva apelando a los criterios de la teoría que previamente acepta. En este escenario, los criterios de evaluación y elección resultarán relativos a cada teoría en un sentido circular que anula la posibilidad de elección racional, desembocando en el riesgo del relativismo epistémico. Esta posibilidad dio lugar a que conforme avanzaba la segunda mitad del siglo XX, se fuera imponiendo la presunción de que propuestas como las de Kuhn o Feyerabend (1994) conducían al relativismo y a la negación exacerbada de la racionalidad científica. En este contexto, buena parte del debate suscitado a partir de los años 60's parecía partir de la premisa implícita de que la única forma de preservar la noción de racionalidad era a través de una concepción formal y universalista, por lo que una vez puesta en crisis esta posibilidad, las diferentes variedades de relativismo e irracionalismo parecían inevitables.

No obstante, en las últimas tres décadas del siglo XX comienza a tomar forma la presunción de que es posible presentar estrategias de defensa de la racionalidad que eviten posiciones relativistas e irracionalistas, y que al mismo tiempo no desemboquen en los compromisos insalvables de las concepciones algorítmicas y universalistas. Es así como, a partir de la obra de pensadores como Hilary Putnam (1988) y Larry Laudan (1986) entre otros, comienza a articularse la idea de que es posible defender otro tipo de racionalidad diferente al que había prevalecido en la primera mitad de siglo, incorporando incluso algunos elementos conceptuales de las obras de Kuhn y Feyerabend en pro de una nueva caracterización de la racionalidad. En este marco toman forma diferentes caracterizaciones informales de la racionalidad que tendrán su desarrollo desde los últimos años del siglo XX hasta la actualidad.

En Razón, Verdad e Historia (1988) —en delante RVH - Putnam defiende que no existen criterios universales para la justificación racional de creencias, sino sólo 
criterios dependientes del contexto y de los sistemas creencias a los que pertenecen. Esto no implica que la elección de criterios de racionalidad sea arbitraria, sino que está sujeta a necesidades de interrelación cognitiva con el entorno. Esta formulación va de la mano con la oposición de Putnam a lo que entiende como concepciones relativistas de la racionalidad: "the fact that the canons of rationality are themselves evolving doesn't mean they don't exist (pace Feyerabend, pace Foucault!), nor does it mean that, in the course of evolution, anything whatsoever might occur." (Putnam 1978, p.169). Consideraciones de esta clase darán lugar a una perspectiva pluralista, de acuerdo a la que es posible defender de forma racional una creencia sin invocar criterios de racionalidad válidos universalmente, pudiendo a su vez negarse la existencia de tales criterios sin derivar en formulaciones que conduzcan al relativismo epistémico. El pluralismo tal como aparece en Putnam implica aceptar que las pautas de racionalidad, en tanto elementos históricamente variables, son internas a los diferentes sistemas o esquemas conceptuales ${ }^{1}$ con los que los sujetos interactúan con el ambiente y producen conocimiento.

Desde una concepción pluralista de la racionalidad, resulta necesario establecer cómo es posible sostener que una creencia es racional si no se cuenta con una noción universal de aceptabilidad racional, en cuanto lo que es racionalmente aceptable depende de la constitución de cada sistema de creencias. Siendo esto así, la defensa de una creencia es relativa a los criterios del sistema desde el que se ha decidido su aceptación, por lo que la misma creencia evaluada desde criterios incompatibles podría declararse no-racional. Una vez que el pluralismo acepta el carácter revisable de los principios de racionalidad, puede suceder que la revisión de un principio que en algún momento lleva a un sujeto a elegir una creencia determinada lo lleve a elegir una creencia lógicamente incompatible u opuesta en un momento posterior. Asimismo, también pude suceder que dos sujetos opten racionalmente por creencias opuestas entre sí, en cuanto las elecciones se realizan privilegiando principios diferentes. En tercer término, es también posible que sea racional sostener una creencia en un momento histórico dado y no sostenerla en un momento histórico posterior. Frente a estos casos y otros análogos, una perspectiva pluralista debería proporcionar un marco conceptual lo suficientemente amplio para abarcar la diversidad y lo suficientemente sólido para identificar de modo los casos de elección racional.

A partir de aquí sostiene Putnam que los principios con que establecemos la racionalidad de nuestras creencias son revisables, en cuanto son producto de la interacción con el entorno. Esto implica que si dos sujetos poseen esquemas conceptuales incompatibles ello responde a que tienen necesidades y propósitos epistémicos diferentes, y en esa medida es posible afirmar que las elecciones de ambos -incompatibles entre sí- son racionales. Desde este modo, la racionalidad informal reconoce la diversidad de contextos epistémicos y la formación de diferentes esquemas conceptuales a partir de ellos. Pero para una caracterización de la racionalidad

Principia 17(3): 419-438 (2013). 
como la defendida por Putnam, es central que en la codependencia entre principios y experiencia sea el mundo empírico el que provea de razones para modificar los principios de aceptabilidad racional. Por esto, cabe pensar que una revisión de principios es racional si es resultado de un cambio de necesidades en relación a la experiencia, y no es racional si no responde a una modificación significativa de la experiencia.

En estas condiciones, cabe preguntarse qué tipo de principios pueden oficiar en un marco de racionalidad informal como instancia de justificación racional de creencias; esto es, qué clase de elementos pueden funcionar como principios de justificación racional una vez que se abandona una concepción algorítmica y universalista de la racionalidad. $\mathrm{O}$ en términos más amplios, qué concepción de la racionalidad puede ofrecerse bajo una perspectiva que admite la variabilidad histórica de los criterios de justificación de creencias, así como su dependencia respecto de los esquemas conceptuales a los que pertenecen.

\section{Justificación racional de creencias}

En Las mil caras del realismo —en delante $M C R$ - Putnam afirma que quien pretende que sus creencias son racionales y a la vez las protege de la crítica racional está incurriendo en una actitud irracional,

No puede haber nada como una afirmación que sea verdadera, a menos que exista la posibilidad de una comunidad de comprobadores, o en todo caso, de críticos. El resultado es que si soy una persona racional, en el sentido de tener el propósito de realizar afirmaciones que sean verdaderas humanamente hablando, es decir, que puedan soportar la crítica ahora y en el futuro, entonces estoy comprometido con la idea de una posible comunidad de investigadores ... Una comunidad que sea competente para determinar la verdad y la falsedad debe ser tal que, por ejemplo, cualquiera en esa comunidad pueda criticar lo que se exponga sabiendo que su crítica será escuchada; si algunas críticas simplemente no son oídas, entonces la posibilidad de algún tipo irracional de 'protección de la creencia' asoma su fea cabeza .... (Putnam 1994, p.114)

Aquí como en otros lugares de su obra, Putnam se nutre del pensamiento de Dewey, quien había definido al fanatismo como la defensa de principios absolutos impasibles de ser puestos en duda o sometidos a discusión (Dewey 1961, p.18-9). De la formulación de Putnam podría extraerse el siguiente principio de racionalidad: no es racional sustraer a la crítica las creencias que se postulan como racionales. En este contexto, criticar o revisar una creencia significa indagar bajo qué condiciones podría ser declarada falsa, o podrían modificarse los conceptos que la componen. Así, una creencia que está excluida de la crítica es aquella cuyas eventuales condiciones de falsedad no pueden ser concebidas o indagadas por el sujeto que la defiende. En estos términos, la afirmación de que no es racional proteger de la crítica las creencias que

Principia 17(3): 419-438 (2013). 
se postulan racionales, implica sólo establecer que no es racional sustraer de la crítica cierta clase de creencias, pero no involucra afirmaciones más específicas acerca de cuáles son las condiciones de la crítica, ni las condiciones por las que una creencia podría llegar a postularse como racional. Esto significa que puede haber sujetos con diferentes esquemas conceptuales y diferentes nociones de racionalidad, y puede decirse que su actitud no será racional si sustraen alguna creencia que pretenden racional a los códigos de racionalidad de su propio esquema.

Se trata de un principio general pero indeterminado, que sólo adquiere contenido al asociarse a pautas de racionalidad de esquemas conceptuales concretos. Posee un tipo de generalidad análogo al que Putnam atribuye al enunciado la ciencia busca la verdad (1988, p.132), ya que este, tomado como un principio de definición de los fines de la ciencia, no adquiere contenido hasta que se asocia a pautas de aceptabilidad bajo las que los científicos llevan a cabo sus investigaciones. Y siendo estas pautas históricamente variables y relativas a cada esquema, un enunciado de este tipo sólo establecería una clase de procedimiento para la justificación racional de creencias, pero no un algoritmo independiente de los contextos de aplicación.

Sin embargo, ya desde mediados de la década de los 70's Putnam había insistido en que hay enunciados no-revisables, y en esa medida sí están sustraídos de la crítica, preguntándose bajo qué condiciones podría aceptarse un enunciado como todos los enunciados son verdaderos (1988, p.90). Esta postura se remonta a trabajos como “There is at least one a priori truth" (1978), en los que Putnam intentará defender contra Quine (1984) - que no todos los enunciados de un sistema de conocimiento dado son revisables, en cuanto existen principios que por sus características intrínsecas pueden considerarse como a priori, y por tanto racionalmente inmunes a la revisión. Así toma cuerpo la idea de que un principio a priori es racionalmente defendible en toda circunstancia, por lo que determinar si existen o no tales principios se vuelve central para cualquier teoría de la racionalidad.

A estos efectos Putnam introduce el "principio débil de no contradicción" —no todo enunciado es verdadero y falso- como un caso de a priori racionalmente aceptable para toda circunstancia (Putnam, 1978). Postula la aprioridad absoluta del principio, presentándolo como una condición general del pensamiento y como base de aspectos específicos del conocimiento, como la producción de informes observacionales y la formulación de predicciones. Sin embargo, las dificultades asociadas a la existencia de principios a priori absolutos conducirán finalmente a restringir su relevancia y su alcance (Putnam 1988, p.90-1), dando lugar a la idea de que los principios a priori admiten ciertos tipos de revisión, y concediendo también que hasta los principios pretendidamente rectores de la racionalidad presentan excepciones. Cabe señalar que para una caracterización informal de la racionalidad resulta problemático defender la existencia de enunciados a priori, entendidos como enunciados que no tienen circunstancias de revisión racional, ya que su status racional

Principia 17(3): 419-438 (2013). 
resultaría independiente de los contextos epistémicos y de las características históricas de los esquemas conceptuales, teniendo un carácter de universalidad irrestricta incompatible con una perspectiva pluralista. Por esto, es de relieve señalar que el desarrollo de las premisas fundamentales de la racionalidad informal por parte de Putnam en $R V H$, coincide con una definida moderación de la importancia y el status de los principios a priori, que marca un cambio de postura respecto de la defensa de la aprioridad absoluta efectuada en trabajos como "There is at least one a priori truth".

Bajo la premisa de que todo principio de justificación racional funciona como tal en el contexto de un esquema conceptual, cabe indagar bajo qué condiciones es posible la evaluación racional de creencias y la justificación en base a principios informales. Inicialmente, es necesario considerar que existen diferencias entre un principio como (1) No todos los enunciados son verdaderos, y otro como (2) No es racional sustraer a la crítica los enunciados que se consideran racionales, ya que a efectos de la justificación racional, (2) tiene más alcance y fertilidad que (1). Supóngase un sujeto $S$ que debe optar entre las creencias $c_{1} \mathrm{y} c_{2}$, siendo $c_{1}$ la negación de $c_{2}$. En este caso la aplicación de (1) sólo le permite a $S$ saber que no puede elegir simultáneamente $c_{1}$ y $c_{2}$ en cuanto ambas no pueden ser verdaderas, pero no le proporcionaría más elementos para comparar $c_{1}$ con $c_{2}$, por lo que no le permite establecer qué creencia es más racional. Sin embargo, si $S$ aplica (2), obtiene que $c_{1}$ y $c_{2}$ deben ser evaluadas en igualdad de condiciones, en cuanto ninguna puede — racionalmente- sustraerse a la crítica conforme a los parámetros de racionalidad que $S$ acepta. En un marco de racionalidad informal, no es seguro que por este procedimiento derivado de (2) $S$ logre determinar qué creencia es más racional, ya que si esto sucediera (2) tendría el status de un principio algorítmico. Pero sí es posible que a través de (2) $S$ logre establecer cuál de las dos creencias es más racional, condición que no se cumple para (1). De este modo, la elección de $S$ sería racional si, después de revisar $c_{1}$ y $c_{2}$ desde las pautas de racionalidad que previamente acepta, elige la creencia que mejor se ajusta con estas pautas, y sería irracional si $S$ elige alguna de las dos creencias sin haberla sometido a revisión conforme a las pautas de racionalidad de su esquema.

También puede suponerse otro sujeto $S^{\prime}$ que proponga evaluar $c_{1}$ y $c_{2}$ en función de un nuevo principio (3). En este caso, la adecuación de (3) también podría ser evaluada, comparando (3) con (2). Si el contenido de (3) fuera es racional no dar motivos de por qué se considera racional una creencia, podría defenderse que (3) no es aceptable como principio de racionalidad. Se podría alegarse que (3) es autocontradictorio, aduciendo que la racionalidad se basa en dar razones de lo que se cree o lo que se hace. Pero si quien interpone (3) insiste en que se puede ser racional sin dar razones, debería o bien proporcionar razones para aceptar su concepción de la racionalidad, o insistir en que su concepción de la racionalidad sea aceptada sin mediar razones. En el primer caso está siendo incoherente con (3); en el segundo 
caso no lo está siendo, pero debilita su posición en cuanto no proporciona ningún elemento para aceptar (3) en lugar de (2). Se podría alegar igualmente que si (3) puede ser aceptado sin razones, también (2) puede serlo. Lo que está involucrado en la evaluación es que quien defiende un principio como (3) no puede sostener con éxito una idea inteligible de la racionalidad y la justificación en sus propios términos. Desde este punto de vista, (3) resulta rechazable como principio de racionalidad no porque no cumpla con un ideal externo de lo que debe ser la racionalidad, sino porque desde un punto de vista interno las propias condiciones que impone el principio resultan inconducentes para su defensa.

Este tipo de estrategia argumentativa es la que toma Putnam en la célebre discusión de la tesis de los cerebros en la cubeta. Frente a quien defiende que somos cerebros en una cubeta, no es posible ofrecer algo tal como una demostración de la existencia del mundo exterior, o una refutación concluyente del escepticismo, sino que la forma de contrarrestar la tesis consiste en evaluar qué consecuencias se siguen de aceptar que somos cerebros en una cubeta, y cómo puede defenderlas quien suscribe la tesis. Si somos cerebros en una cubeta — señala Putnam-, entonces nuestras afirmaciones sobre árboles, muebles y demás objetos, no tienen valor referencial, es decir no refieren a objetos reales, sino a los inputs que llegan a nuestro cerebro creando ilusiones perceptivas. Pero si esto es así, se sigue que podrían no existir los árboles en el exterior de la cubeta, y de todas formas los cerebros en la cubeta seguirían hablando de árboles, porque su emisión de árbol, no refiere a nada en el mundo fuera de la cubeta. Ahora bien, si sus pensamientos y emisiones lingüísticas - ilusorias - no refieren a nada fuera de la cubeta, entonces un cerebro en una cubeta no puede afirmar soy un cerebro en una cubeta, porque la frase no tiene conexión causal con la cubeta y el cerebro del mundo exterior, por lo que sólo puede significar que somos cerebros ilusorios — como los árboles y demás objetos— en una cubeta ilusoria. De modo que si es verdad que somos cerebros en una cubeta, la frase somos cerebros en una cubeta, expresa algo falso, y en esa medida es autocontradictoria (Putnam 1988, cap. 3).

Por otra parte, quien postula que somos un cerebro en una cubeta supone que todo nuestro - presunto- conocimiento del mundo exterior se asienta sobre una ilusión tan perfecta que impide formular con verdad cualquier afirmación, incluso la afirmación de que somos cerebros en una cubeta, por lo que la visión del mundo que defiende resulta incoherente (Putnam 1988, p.137 y ss.). ${ }^{2}$ Se podría contrarrestar esto afirmando que el defensor de los cerebros en la cubeta pueden tener estándares de coherencia diferentes a los nuestros; y en ese caso podrían ser sometidos a revisión, pero el proceso de revisión no podría ser ilimitado ni irrestricto, en cuanto el hecho de entablar un debate y defender un sistema inteligible de creencias impone ciertas constricciones a los interlocutores, por más que partan de esquemas conceptuales diferentes. Quien afirma que somos cerebros en una cubeta puede interponer 
un estándar de coherencia diferente o incompatible con el nuestro, pero no puede — por ej._ afirmar que según su estándar dos enunciados son coherentes entre sí si siendo contrarios pueden ser ambos verdaderos; como tampoco puede decir que un sistema es coherente sólo si contiene enunciados terminados en palabras esdrújulas. No podría decir esto porque ambas respuestas no sólo restan inteligibilidad a la discusión —podríamos preguntarle por qué son incoherentes los sistemas con enunciados terminados en palabras agudas-, sino que - ante todo- no cubrirían las necesidades de conocimiento al que su propio sistema pretende responder.

De esta forma, el planteo de Putnam permite entrever no sólo la manera en que la coherencia funciona como un valor, estipulado y aplicado de forma no algorítmica en la regulación del desacuerdo, sino también como es posible declarar legítimamente un sistema de creencias como incoherente o irracional. La evaluación de la racionalidad de una hipótesis o un principio de justificación de creencias, responde a la fertilidad del principio en juego, y a la medida en que conduce o no a consecuencias incoherentes. Desde esta perspectiva puede afirmarse que (2) es preferible a (3) como principio de racionalidad, en cuanto (3) conduce a consecuencias incoherentes y autocontradictorias, en términos similares a los que permiten rechazar la tesis de los cerebros en la cubeta.

\section{Relatividad conceptual y principios de racionalidad}

En la contraposición de (2) con (3) es posible la distinción entre un principio racionalmente defendible y uno que no lo es, o al menos no parece serlo. Sin embargo, no todos los casos de principios en conflicto pueden resolverse de la misma manera, en cuanto hay escenarios más problemáticos que la oposición entre (2) y (3), que funcionan como restricciones legítimas de (2). Puede suponerse un sujeto $S^{\prime \prime}$, defensor de $c_{2}$, que proponga el principio (4): Bajo ciertas condiciones es racional sustraer ciertas creencias a la crítica, con lo que quedaría abierta la posibilidad de que $S$ opte por $c_{1}$ y $S^{\prime \prime}$ por $c_{2}$. Quien defiende (4) puede aducir que no ve razones puntales para exponer a la crítica determinadas creencias de su esquema conceptual, y que no considera justificado exponerlas a la crítica sólo en nombre de la premisa general de que todas las creencias racionales son revisables. Puede sostener también que es imposible exponer a revisión todas las creencias que componen un esquema conceptual, por lo que necesariamente algunas van a quedar expuestas a la crítica y otras no. El mismo Putnam concede esta posibilidad cuando sostiene que "Any Valuing can become evaluated, although we cannot ever be in a position in which all valuings have been evaluated." (Putnam 1994 p.201). En suma, el sujeto $S^{\prime \prime}$ puede proporcionar una amplia serie de argumentos que no impliquen una contradicción con sus propios términos, una serie de consecuencias inaceptables o una incoherencia con 
el principio general que tomó como punto de partida. Es posible que en este caso no haya forma de establecer que la elección de $S$ es más racional que la de $S^{\prime \prime}$, en cuanto es concebible que (4) resulte para el esquema conceptual de $S^{\prime \prime}$ tan adecuado como (2) resulta para $S$, ya que ambos sujetos pueden mantener el principio inicial y asumir de modo coherente — cada uno dentro de su esquema — las consecuencias que se derivan de su aceptación.

En estos términos, (4) funciona como una restricción de (2), de forma que (4) y (2) son ambos racionalmente elegibles aunque sean incompatibles. Esto representa una dificultad sólo si se parte de que una creencia es racional de modo concluyente o no es racional en absoluto; pero en una concepción pluralista no sólo no se acepta esta premisa, sino que no se requiere que los principios de racionalidad sean elegibles en toda circunstancia o no puedan coexistir con principios rivales. Por esto puede decirse que principios como (2) y (4) resultan altamente funcionales como principios de racionalidad, no obstante poseen un grado de generalidad restringida, en cuanto admiten restricciones derivadas de la interposición de otros principios. Esta afirmación también responde a una idea inspirada en el pensamiento de Dewey, a saber, que una discusión entre posiciones en conflicto - ya se trate de cuestiones éticas o empíricas- puede ser estrictamente racional sin que ello arroje un resultado concluyente acerca de cuál de las posturas en conflicto está más justificada:

If you and i disagree in our "values" a resolution need not take the form of a universalistic principie that you and $i$ and all other persons can accept as valid. If the value disagreement concerns only our separate lives, then the apparent disagreement may be resolvable by simply relativizing the judgements in question ... A similar situation arises in exact science; when we discovered that the simultaneity judgements of different observers could be incompatible, we resolved the resulting problem by adopting a theory according to which such judgements are relative to the state of motion of the observer. (Putnam 1994, p.214)

En la contraposición de (2) con (4) dos sujetos optan por creencias opuestas resultando racionales las elecciones de ambos. Esto no implica que los principios no sean comparables, implica que no existe algoritmo de comparación, y que en algunos casos las comparaciones son posibles y en otros no. Lo que resulta fundamental en una concepción informal es la exclusión dos escenarios: la posibilidad de decidir de modo concluyente cuál de los principios en juego es el adecuado, y la imposibilidad de tomar cualquier decisión sobre su adecuación. La racionalidad informal conlleva la negación de principios universales para la justificación de creencias, pero no impide la defensa de otra clase de principios. A partir de aquí es posible identificar algunas características definitorias de los principios informales de racionalidad. Se trata de principios relativos, en cuanto dependientes de necesidades históricamente variables; indeterminados, en cuanto no establecen contenidos condiciones unívocas

Principia 17(3): 419-438 (2013). 
de aplicación; ${ }^{3}$ y de generalidad restringida, en cuanto admiten restricciones derivadas de principios rivales. Una vez que la revisión de los principios es amplia pero no ilimitada, resulta posible evaluar la adecuación y la fertilidad de principios en conflicto, si bien el resultado de la evaluación no es concluyente.

Para dar una formulación más precisa a la afirmación de que los principios de la racionalidad informal son relativos, es útil recurrir a la noción putnamiana de relatividad conceptual, entendida como la posibilidad de coexistencia de dos o más esquemas conceptuales incompatibles entre sí. La relatividad conceptual se distingue del relativismo en cuanto no implica que cualquier esquema sea elegible frente a cualquier otro, así como tampoco que no haya modo de fundamentar las elecciones. Implica solamente que hay más de una elección racionalmente posible (Putnam 1987, p.61 y ss.). Putnam especifica este punto con un conocido ejemplo. Supóngase un mundo carnapiano compuesto por los individuos $X 1, X 2$ y $X 3$, y pregúntese cuántos objetos componen ese mundo. Una primera respuesta es que habiendo sólo tres individuos, hay sólo tres objetos. Se trata de una respuesta aceptable, pero el problema es si se trata de la única respuesta legítima. Una segunda posibilidad podría ser partir de la premisa mereológica de que una suma constituye un objeto diferente de los términos que la componen, de modo que el mundo contiene siete y no tres objetos, dados por los iniciales $X 1, X 2$ y $X 3$, más las cuatro sumas posibles entre ellos. Podemos adoptar tanto el sistema carnapiano como el sistema mereológico, y la cantidad de objetos que identifiquemos en el mundo va a ser relativa al esquema conceptual que hayamos elegido. Los conceptos de ambos sistemas tienen un valor relativo en cuanto son dependientes de la estructura del sistema elegido, pero no en cuanto sean resultado nada más que de una convención o una decisión. "Nuestros conceptos puede ser relativos a una cultura, pero de aquí no se sigue que la verdad o la falsedad de cualquier cosa que digamos usando esos conceptos sea simplemente decidida por la cultura." (Putnam 1987, p.62-4). Así entendida, la noción de relatividad conceptual sirve antes como una estipulación de los límites de la justificación y el ejercicio racional, que como una flexibilización de la noción de racionalidad, en cuanto si bien obliga a aceptar que en situaciones de conflicto hay más de una elección racional posible —en el ejemplo pueden ser tres objetos, siete objetos, etc.- mantiene la premisa racionalista de que no todas las elecciones son posibles y que no todo es sólo cuestión de elección.

Desde una orientación muy diferente a la de Putnam, pero que no deja de tener puntos de convergencia, van Fraassen afirmó que "Una epistemología completa debe investigar cuidadosamente las condiciones de racionalidad para la aceptación de las conclusiones que van más allá de la evidencia que uno tiene. Aquello que, creo, no puede proporcionarnos (...), son reglas que nos obliguen racionalmente a tomar esas decisiones epistemológicas." (van Fraassen 1996, p.98). Este tránsito de las reglas de racionalidad a las condiciones de racionalidad puede entenderse como el tránsito

Principia 17(3): 419-438 (2013). 
desde la racionalidad formal algorítmica a la racionalidad informal pluralista, bajo la cual la tarea de una teoría de la racionalidad es reconstruir las condiciones bajo las que los sujetos pueden declarar como racionales ciertas creencias, sin esperar que la reconstrucción arroje como resultado un conjunto de condiciones necesarias y suficientes para toda creencia racional.

\section{Racionalidad y revisabilidad}

A pesar de la imagen ciertamente estable que a principios de los 80's la filosofía de Putnam arroja sobre las características básicas de la racionalidad, no deja de ser llamativo el que Putnam tome en $M C R$ una perspectiva compatible con el principio No es racional sustraer a crítica los enunciados que se consideran racionales (2), mientras que en $R V H$ rechaza el principio quinenano según el que Ningún enunciado es inmune a la revisión (5). Frente al aire de familia que a primera vista presentan ambos principios, cabe preguntarse si bajo un marco de racionalidad informal es posible aceptar el primero y rechazar el segundo. Esto debería ser posible si la postura de Putnam entre $R V H$ y $M C R$ fuera estrictamente consecuente, mientras que si el aceptar (2) implica aceptar (5) cabría pensar que en MCR Putnam habría reformulado parcialmente su concepción de la racionalidad, a efectos de dar cabida a alternativas rechazadas en $R V H$.

Una estrategia posible para mostrar en qué medida se trata de principios diferentes, tiene que ver con su ámbito de aplicación; mientras que (5) establece que no hay enunciados inmunes a la revisión, por lo que no es racional sustraer a la crítica ningún enunciado, (2) establece que no es racional sustraer a la crítica solamente los enunciados que de antemano se han declarado racionales. De este modo, un sujeto podría lícitamente sustraer a la crítica una o más creencias correspondientes a su esquema conceptual por el hecho de no conferirles un status de creencias racionales, y de esta forma estaría aceptando (2) y no (5), ya que de acuerdo a (5) esta posibilidad queda vedada, en cuanto el sujeto estaría obligado a someter a revisión cualquier creencia que forme parte de su sistema.

Hasta aquí resulta consistente atribuir a Putnam la negación de (5) y la afirmación de (2), sin embargo la cuestión presenta aún alguna dificultad. Como ya se mencionó, Putnam presentó en $R V H$ el caso de (1) No todos los enunciados son verdaderos como caso contrario a (5), en cuanto no parece plausible pensar en condiciones bajo las que sea lícito aceptar que todos los enunciados son verdaderos. Pero el problema aquí radica en que si se acepta que todos los enunciados racionales son pasibles de revisión (2), parece necesario aceptar que un enunciado como (1) es pasible de revisión, salvo que se quiera tomar la alternativa poco prometedora de excluirlo del dominio de la racionalidad. En estos términos, si bien lo afirma-

Principia 17(3): 419-438 (2013). 
do por Putnam en $M C R$ no conduce automáticamente a la aceptación del principio quineano de que todos los enunciados son revisables, puede sí decirse que la postura de Putnam en $M C R$ implica una reformulación parcial de la caracterización de la racionalidad informal introducida en $R V H$; reformulación que deja más espacio a la posibilidad de revisión de principios y menos margen aún para la defensa de elementos a priori o de principios intrínsecamente irrevisables.

No obstante, aún es posible preguntarse, ¿bajo qué condiciones podría ser revisable una afirmación como no todos los enunciados son verdaderos? ${ }^{4}$ Quizás la forma de compatibilizar (1) con (2), o mejor dicho de incluir (1) en el radio de acción de (2) - a saber el de los enunciados revisables-, no consiste en especificar bajo qué condiciones estaríamos dispuestos a revisar nuestra creencia de que no todos los enunciados son verdaderos, sino en aceptar que en cuanto la consideramos una creencia racional, estaríamos dispuestos a revisarla si eventualmente se dieran las condiciones que justificaran hacerlo, aún cuando actualmente no estemos en condiciones de especificarlas. En esta línea, Putnam señala que el principio de no contradicción quizás sea el único enunciado pasible de ser probado a toda persona racional (1987, p.134); lo que también podría valer para algunos principios matemáticos como todo número tiene un sucesor, previamente considerados como constitutivos de la racionalidad (Putnam 1977, p.10). Podemos considerar estos principios como fundamentales para nuestras pautas de racionalidad, ya que no concebimos condiciones en las que puedan ser racionalmente negados, pero podemos al mismo tiempo establecer que estaríamos dispuestos a revisarlos si se dieran las condiciones adecuadas. Hasta aquí, el cumplimiento de (2) parece requerir antes la disposición a la revisión que la declaración de las condiciones de posibilidad de la revisión, por lo que (1) sería tan revisable como cualquier ley lógica o cualquier axioma matemático. Por el contrario, si se pretende concederle a alguno de estos principios un carácter a priori más allá de toda revisión, es necesario negar o al menos moderar el principio (2).

Esta disyuntiva puede despejarse apuntando que la imagen general de la racionalidad presentada por Putnam se compagina de buen grado con la idea de que, si bien no existen enunciados intrínsecamente irrevisables, no es posible exponer a revisión racional todos los enunciados de un esquema conceptual al mismo tiempo, en cuanto el proceso mismo de exponer ciertos enunciados a la revisión implica preservar otros. Señala Putnam "There are some statements which we can only verify by failing to verify other statements. This is so a matter of logic (for example, if we verify 'in the limit of inquiry' that no one ever will verify or falsify $p$, where $p$ is any statement which has a truth value, then we cannot decide the truth of $p$ itself...)." (Putnam 1990, p.viii). Si exponemos a revisión y confirmamos el enunciado $p$ no es verificable ni falsable, no podemos revisar confirmar el enunciado p. Del mismo modo, la confirmación de un enunciado como la posición de Saturno en el momento $m$ era $x$, sólo puede ser confirmado preservando de crítica las teorías ópticas y físicas 
que explican el funcionamiento del telescopio que permite obtener datos sobre posiciones planetarias. Tanto en este ejemplo como en el de Putnam, puede señalarse que un cambio en el diseño experimental cambiaría las condiciones de exposición a la crítica: podemos efectivamente tratar de confirmar o falsar $p$, y en esa medida lo estamos sometiendo a crítica —independientemente deque tengamos éxito o no-, del mismo modo que podemos hacer experimentos para perfeccionar telescopios, testear su falibilidad o poner a prueba leyes de la óptica.

Puesto en estos términos, la racionalidad de preservar ciertos enunciados de la crítica parece revestir un carácter estrictamente metodológico: en función de lo que queremos confirmar - posiciones planetarias o leyes ópticas-, exponemos ciertos enunciados a revisión y preservamos otros. Sin embargo, puede haber casos en los que la preservación de la revisión tiene algo más que este carácter metodológico, dando cuenta de un límite epistémico. Cuando un científico confirma un enunciado como $X$ es la causa de $Y$, la confirmación sólo tiene sentido si se preserva de crítica algún enunciado previo como en la naturaleza existen relaciones causales. ${ }^{5}$ Del mismo modo, la producción de informes observacionales en ciencia supone preservar de crítica ciertos principios generales sobre la naturaleza del conocimiento, tales como la información empírica bajo condiciones controladas es fiable, la naturaleza es cognoscible, o la matemática es una herramienta adecuada para conocer del mundo empírico. La particularidad de este tipo de principios radica en que permanecen profundamente arraigados en los procesos de producción de conocimiento dados al interior de un esquema, de tal modo que los sujetos que los aceptan no pueden concebir condiciones en las que serían falsos.

Este tipo de principios da cuenta de los límites de la racionalidad, y de las condiciones de producción de conocimiento bajo esquemas conceptuales en contextos históricos específicos, permitiendo una mejor comprensión de la oposición de Putnam a la tesis quineana de que todos los enunciados son revisables. El enunciado las órbitas planetarias son circulares, permaneció como irrevisable desde la antigüedad griega hasta Kepler, en cuanto los astrónomos no podían concebir condiciones en las que pudiera ser falso. Del mismo modo, difícilmente los físicos actuales podrían concebir una teoría física que no utilizara matemáticas, o que comprendiera el espacio en términos no-relativistas. Durante un tiempo Putnam adjudicó a este tipo de principios un status a priori contextual (Putnam 1976; 1983) señalando que en contextos históricos concretos detentan un status a priori, en cuanto no tienen condiciones de revisión racional, no obstante pueden con el paso del tiempo resultar expuestos a la crítica como consecuencia del advenimiento de una teoría o una serie de principios rivales, que precipitan la pérdida del status a priori. Tal es el caso de los axiomas de Euclides, que durante siglos fueron considerados irrevisables, hasta que el cambio conceptual operado con las geometrías no euclídeas generó una modificación en su status, habilitando posibilidades de revisión vedadas previamente.

Principia 17(3): 419-438 (2013). 
La tesis de que algunos principios pueden ser racionalmente preservados de la crítica en ciertas condiciones históricas implica que la revisabilidad de los enunciados que componen un esquema conceptual es indefinida desde el punto de vista histórico, ya que bajo condiciones históricas concretas cualquier enunciado puede volverse revisable, pero no es posible que todos los enunciados de una teoría o un esquema conceptual sean revisables al mismo tiempo, en la medida en que todo sistema requiere de enunciados irrevisables que marcan sus límites y sus posibilidades de generación de conocimiento. Puede entonces decirse que no todos los enunciados de un sistema de conocimiento son revisables actualmente, pero que todos son revisables potencialmente, en función de los cambios dados en las condiciones epistémicas. No obstante, los procesos de revisión siempre supondrán exponer a la crítica algunos enunciados preservando otros, por lo que puede también afirmarse que los enunciados de un sistema de conocimiento no son simultáneamente revisables. Por esto, bajo una concepción pluralista e informal de la racionalidad queda cancelada tanto la afirmación de principios de justificación universales e invariantes, como también la posibilidad de someter a crítica uno-a-uno todos los enunciados que componen un sistema de conocimiento. Del mismo modo que una característica estructural de la racionalidad parece ser la imposibilidad de resolver de modo concluyente todos los desacuerdos racionales, una característica de tener creencias y defenderlas como racionales pasa por exponer algunas a la crítica y preservar otras de toda revisión.

\section{La verdad como aceptabilidad racional idealizada}

Esta caracterización de la racionalidad se sostiene en la concepción de la verdad como aceptabilidad racional idealizada desarrollada por Putnam, de acuerdo a la que la declaración concluyente de la verdad de un enunciado, sólo podría darse suponiendo un escenario en que un sujeto cuenta con la información perfecta para el establecimiento de tal verdad. Esto es, toda la información relevante acerca de condiciones iniciales, creencias previas, factores de incidencia, posibilidades de error, etc. "Truth, in the only sense in which we have a vital and working notion of it, is rational acceptability (or, rather, rational acceptability under sufficiently good epistemic conditions; and which conditions are epistemically better or worse is relative to the type of discourse in just the way rational acceptability itself is)." (Putnam 1981, p.231) En condiciones idealizadas de aceptabilidad racional, un enunciado podría ser declarado verdadero, o intrínsecamente aceptable en términos racionales, pero la especificación de las condiciones reales siempre se realiza en el lenguaje y bajo los criterios de un esquema conceptual determinado, por lo que la especificación de las condiciones reales siempre va a ser relativa a un marco lingüístico.

La caracterización de Putnam deriva así en que "La verdad es una idealización

Principia 17(3): 419-438 (2013). 
de la aceptabilidad racional. Hablamos como si hubiera tales cosas como condiciones epistemológicas ideales, y llamamos "verdadero" a un enunciado que estaría justificado bajo tales condiciones. Las condiciones epistemológicamente ideales son como las superficies sin rozamiento: en realidad no podemos obtener condiciones epistemológicamente ideales, ni siquiera tener la certeza de que nos hemos aproximado suficientemente a ellas." (Putnam 1988, p.65) En condiciones epistémicas ideales, un enunciado verdadero es aquél que ha sido confirmado en base a toda la información relevante. En condiciones epistémicas reales, la confirmación de un enunciado y su declaración de verdad siempre se realizan bajo los criterios de un esquema conceptual, dentro de los límites que este permite, y con una cantidad de información parcial e incompleta. Desde este punto de vista no es posible determinar qué tan óptimas son las condiciones epistémicas que justifican una creencia desde fuera del marco de un esquema conceptual. Toda evaluación de las condiciones de justificación se efectúan en un lenguaje dado, que responde a criterios específicos de racionalidad, y en función de ello se afirma que una creencia es más o menos racional, pero no puede hacerse una evaluación semejante desde ningún lugar, o prescindiendo que se trata de una evaluación desde un esquema

Señala también Putnam "Even if what i were offering were a defínition of truth, the point that it makes about truth apeares within whatever type of language we are talking about; one cannot say what are good or better or worse epistemic conditíons in quantum mechanics without using the language of quantum mechanics; one cannot say what are good or better or worse epistemic situations in moral discourse without using moral language; one cannot say what are good or berter or worse epistemic situations in commonsense material object discourse without using material object language." (1990, p.viii-ix) Esta delimitación lingüística y conceptual de nociones como la de verdad, justificación y razón respecto de la estructura interna de cada esquema conceptual, hace que la declaración de verdad bajo condiciones epistémicas reales resulte necesariamente falible y revisable, y la afirmación de que un enunciado es verdadero pueda siempre modificarse como resultado de la introducción de información no considerada inicialmente, o de modificaciones significativas en el marco conceptual.

Esta concepción de la verdad se articula en una perspectiva falibilista con consecuencias en la teoría de la racionalidad. Si la aceptabilidad racional de un enunciado sólo puede establecerse en condiciones epistémicas ideales, se sigue que en condiciones epistémicas reales ningún enunciado es necesariamente racional o intrínsecamente aceptable en términos racionales. La racionalidad de cualquier enunciado está dada por las condiciones epistémicas concretas en que se postula su carácter racional, siendo este carácter revisable a la luz de nueva información o de cambios en las condiciones. Esta concepción de la verdad permite afirmar que en un marco de racionalidad informal un enunciado puede ser racional bajo ciertas circunstan-

Principia 17(3): 419-438 (2013). 
cias y no serlo en otras diferentes, así como también permite afirmar que — siempre bajo condiciones epistémicas concretas- puede ser racional tanto la defensa de un enunciado como la defensa de su negación.

Cabe señalar que este último punto no implica una apertura al relativismo epistémico ni a la revisabilidad indefinida de los principios de racionalidad. Esto en cuanto Putnam insiste en la 'convergencia' de la verdad, "es de esperar que la verdad sea estable o "convergente"; si tanto un enunciado como su negación pueden ser "justificados", 6 no tiene sentido pensar que tal enunciado posee un valor de verdad, por mucho que las condiciones fueran tan ideales como uno soñase alcanzar." (Putnam 1988, p.65) A partir de aquí pude pensarse que en un caso de imposibilidad de elegir entre principios de racionalidad opuestos incompatibles - como en los principios (2) y (4) revisados-, la imposibilidad de la decisión responde a características de las condiciones en que se desarrolla el debate, y no a que la imposibilidad de decidir sea un rasgo intrínseco de las disputas racionales. Del mismo modo que bajo condiciones epistémicas ideales podría establecerse de modo concluyente la verdad de cualquier enunciado y la falsedad de su negación, sería también posible determinar de modo unívoco la racionalidad de un principio y la no racionalidad de otros principios incompatibles, operación que no es posible en condiciones epistémicas reales.

\section{Conclusión}

Parece ser una alternativa poco prometedora el defender que una teoría satisfactoria de la racionalidad es una teoría que nos permite resolver todos los desacuerdos racionales, ya que la ausencia de una teoría tal desemboca en un desencanto respecto de la racionalidad que conduce fácilmente al irracionalismo. Si se concede esto, entonces puede aceptarse una caracterización de la racionalidad que postule que - en condiciones epistémicas concretas - un enunciado puede estar igual de racionalmente justificado que su negación, y que no todos los desacuerdos racionales son resolubles. En estos términos, la caracterización de la racionalidad ofrecida por Putnam conlleva la negación de principios universales y algorítmicos para la justificación racional de creencias, pero esta limitación no impide la defensa de otra clase de principios de racionalidad. La racionalidad informal admite principios relativos, en la medida en que son dependientes de necesidades cognitivas históricamente variables, y permanecen asociados a esquemas conceptuales. Constituyen principios de generalidad formal, en cuanto son revisables a la luz de principios rivales igualmente fértiles o adecuados y en cuanto no establecen contenidos ni condiciones concretas de aplicación.

Esto supone que una vez que la revisión de los principios es amplia pero no ilimi-

Principia 17(3): 419-438 (2013). 
tada, resulta posible evaluar la adecuación y la fertilidad de principios en conflicto, si bien el resultado de la evaluación no es necesariamente unívoco ni concluyente. En estos términos, la posibilidad de revisión racional puede ampliarse hasta abarcar principios cuyas condiciones de revisión no resultan actualmente concebibles. Estos últimos tiene un status particular, en cuanto permanecen arraigados en los esquemas conceptuales a los que pertenecen, de modo que los sujetos que los aceptan no pueden concebir condiciones bajo las que resultarían falsos. El status - contextualmente a priori- de estos principios, permite postular que una característica intrínseca de tener creencias racionales consiste es exponer algunas de ellas a la revisión, preservando otras que presentan un status diferente a las que resultan revisables.

La caracterización putnamiana de la verdad como aceptabilidad racional idealizada implica que la justificación y la verdad de un enunciado es siempre revisable, en cuanto producto de condiciones epistémicos concretas. No obstante, esto no quiere decir que todas las creencias que componen un esquema conceptual puedan revisarse al mismo tiempo, sino que bajo diferentes condiciones históricas todas pueden llegar a ser revisables, aunque no conjuntamente. Por último, cabe señalar que esta imagen de la racionalidad no deja de revestir cierta tensión con los desarrollos de Putnam en defensa de los enunciados a priori, así como con su crítica a Quine. Cabría pensar que la forma más interesante de reconstruir y encausar esta tensión es optando por una lectura de la racionalidad informal putnamiana que modere las pretensiones de identificar principios absolutamente a priori o intrínsecamente racionales. Esto resulta posible aún concediendo que en un esquema conceptual dado existen enunciados sustraídos de la crítica, pero que ello no responde a sus características intrínsecas sino a las características históricas del esquema conceptual al que pertenecen, y a las necesidades cognitivas de los sujetos que lo utilizan.

\section{References}

Ben-Menahem, Y. 2005. Putnam on skepticism. In: Y. Ben-Menahem (ed.) Hilary Putnam. Cambridge: Cambridge University Press, p.125-55.

Brueckner, A. 1986. Brains in a Vat. Journal of Philosophy 83(3): 148-67. 1992. If I Am a Brain in a Vat, Then I Am Not a Brain in a Vat. Mind 101(401): 123-28.

Davidson, D. 1974. De la idea misma de un esquema conceptual. In D. Davidson: De la verdad y de la interpretación, Barcelona: Gedisa, 1990.

Dewey, J. 1961. El hombre y sus problemas. Buenos Aires: Paidós.

Feyerabend, P. 1994. Contra el método. Barcelona: Planeta-Agostini.

Kuhn, T. 2004. La estructura de las revoluciones científicas. Mexico: FCE.

Laudan, L. 1986. El progreso y sus problemas. Hacia una teoría del progreso científico. Madrid: Encuentro.

Principia 17(3): 419-438 (2013). 
Melogno, P. 2012. Escepticismo y aprioridad contextual. In: C. Caorsi; R. Navia; P. Melogno (comps.) Actas del $1^{\circ}$ Congreso de la SFU, Sociedad Filosófica del Uruguay. Montevideo, p.265-74.

Müller, O. 2001. Does Putnam's argument beg the question against the skeptic? Bad news for radical skepticism. Erkenntnis 54: 299-320.

Navia, R. 1999. Verdade, racionalidade e relativismo em H. Putnam. Porto Alegre: EDIPUCRS. Putnam, H. 1976. Two dogmas revisited. In H. Putnam: Realism and Reason, Philosophical Papers vol. 3. Cambridge, MA: Cambridge University Press, 1983, p.87-97.

- 1977. Models and Reality. In H. Putnam: Realism and Reason, Philosophical Papers vol. 3. Cambridge, MA: Cambridge University Press, 1983, p.1-25.

- 1978. There is at least one a priori truth. Erkenntnis 13(1): 153-70.

- 1981. Beyond historicism. In H. Putnam: Realism and Reason, Philosophical Papers vol. 3. Cambridge, MA: Cambridge University Press, 1983, 287-303.

-1983. Lo analítico y lo sintético. Mexico: UNAM.

- 1988. Razón, verdad e historia. Madrid: Tecnos.

- 1990. Preface. In H. Putnam: Realism with a Human Face. Cambridge, MA: Harvard University Press, 1990, p.vii-xi.

—. 1994. Las mil caras del realismo. Barcelona: Paidós; I.C.E.-U.A.B.

Putnam, H.; Putnam, R. A. 1994. Dewey's logic: epistemology as hypothesis. In: Words and life. Cambridge, MA: Harvard University Press, p.198-220.

Quine, W. 1984. Dos dogmas del empirismo. In W. Quine: Desde un punto de vista lógico. Buenos Aires: Orbis, p.49-81.

van Fraassen, B. 1996. La imagen científica. Mexico: Paidós.

Pablo Melogno

Facultad de Información y Comunicación

Universidad de la República Emilio Frugoni 1427 CP 11200

Montevideo

URUGUAY

www.fic.edu.uy

pmelogno@gmail.com

Resumo. Em Razão, Verdade e História, Putnam caracteriza a racionalidade informal como uma alternativa que permite superar os limites das concepções formalistas da racionalidade. Inicialmente se revisa a caracterização de Putnam, buscando estabelecer qual o tipo de enunciados que pode funcionar como princípio de racionalidade em um marco informal. Se defende que, mesmo que Putnam negue todo princípio universal de racionalidade, admite princípios relativos, gerais e indeterminados, que permanecem sujeitos a necessidade de interrelação cognitiva com o entorno. Se mostra que este tipo de princípios admite exceções, não apresenta conteúdos determinados e não proveem resultados concludentes; todavia, isso não impede a avaliação de princípios em conflito. Se dois sujeitos tem diferentes esquemas conceituais, isso se deve ao fato de terem diferentes propósitos cognitivos, e nesta medida se pode afirmar que as escolhas de ambos são racionais. A partir daí se defende a possibilidade de revisão de princípios cuja negação resulta inconcebível. Finalmente, se afirma que tanto

Principia 17(3): 419-438 (2013). 
expor como preservar crenças da controvérsia constituem elementos históricos que devem ser contemplados em uma concepção informal.

Palavras-chave: Putnam; racionalidade informal; esquemas conceituais; justificação.

\section{Notas}

${ }^{1}$ No se expondrá aquí la noción de esquema conceptual, para cuya presentación puede revisarse Putnam (1988, caps. 3-4). Es de referencia también la crítica clásica de Davidson (1974), y para una revisión detallada puede consultarse Navia (1999).

${ }^{2}$ Los problemas relacionados con el argumento de los cerebros en la cubeta han generado un campo específico de tratamiento dentro de la literatura, cuya vastedad no pretendemos cubrir aquí, en tanto nuestro propósito es solamente ilustrar a través del argumento el tipo de estrategia — basada en la coherencia interna - que toma Putnam para contrarrestar hipótesis que considera irracionales. Para un tratamiento del tema pueden consultarse los trabajos clásicos de Brueckner (1986, 1992), y los más recientes de Müller (2001) y Ben-Menahem (2005).

${ }^{3}$ Algunas formulaciones afines a esta caracterización pueden encontrase ya en períodos tempranos de la obra de Putnam, bastante anteriores a $R V H$. En "Lo analítico y lo sintético" de 1962, afirma que el principio quineano Permítase que todo enunciado pueda sujetarse a revisión resulta insuficiente como regla algorítmica de elección racional, en cuanto debe complementarse con reglas que establezcan qué tipo de revisiones son necesarias, y bajo que procedimientos hay que efectuarlas. Y son justamente variables como los procedimientos, las creencias que se decida revisar en cada caso, y el resultado de la revisión, lo que variará en función de los estándares y códigos aceptados en el momento de la aplicación del principio (Putnam 1962, p.47).

${ }^{4}$ Hemos ofrecido un tratamiento más detallado de este problema en Melogno (2012).

${ }^{5}$ No es relevante que se trate de relaciones causales o de otra clase. El caso sería el mismo para un científico que verificara un enunciado como la frecuencia de $X$ es $Y$, lo que sólo podría hacerse suponiendo un enunciado como en la naturaleza existen regularidades estadísticas, y lo mismo puede decirse de un científico que verifique un enunciado en términos probabilísticos, o similares.

${ }^{6}$ Es decir si fuera posible justificar ambos en condiciones epistémicas ideales (nota del autor).

Principia 17(3): 419-438 (2013). 